\title{
The Evolution of Terrestrial-Type Planets
}

\author{
Don L. Anderson
}

\begin{abstract}
The present internal state of the earth and the age of various events such as core formation and continental formation can be used to place fairly strong constraints on the early history of the earth and, presumably, the other terrestrial planets. The present internal configuration of the earth shows that it is an extremely differentiated body, and the ages of surface rocks indicate that this differentiation took place early in the history of the earth. The gravitational energy associated with the formation of the earth and the thermal energy of core formation are the primary sources of energy for the melting and subsequent gravitational separation of the earth into a core, a crust, a chemically inhomogeneous mantle, and an atmosphere and hydrosphere. The larger the planet the more likely it is to be extensively differentiated and outgassed. The earth probably accreted in $10^{3}$ years or less. This causes the initial temperatures to be high and results in core formation to be essentially simultaneous with accretion. Mars can be accreted without differentiation and with minor outgassing.
\end{abstract}

\section{Introduction}

Geologists have long recognized that the study of rocks and of surface deformations provide important clues to processes operating in the past and operating at great depth in the earth. A small but vigorous school of geologists, geochemists, and geophysicists is concerned with present and past chemical and physical interactions between the crust, the mantle, and even the core. It is now widely recognized that basalts are primitive crustal material and are differentiates of the upper mantle and represent the lighter, low-melting fraction of this part of the mantle. Several other, more refractory rock types have been identified, which apparently formed in the upper mantle and were brought to the surface by solid ejection or intrusion. In the last 20 years, it has also been recognized that the composition and evolution of the oceans and atmosphere are also intimately related to processes operating in the deep interior. The study of the composition of the atmosphere, the hydrosphere, and the crust and inferences regarding the composition of the parent mantle ultimately takes one back to questions of the origin of the earth and the solar system. Solar and planetary astronomers, cosmologists, geologists, geochemists, and geophysicists are all studying parts of the same problem, and to reconstruct the origin and evolution of the earth, or of the other planets, requires input from all these disciplines. The processes and time scales of outgassing and differentiation of the crust-mantle-core, geophysical and geochemical phenomena, are not independent of the process and time scale of planetary accretion, a cos-

The author is with the Division of Geological Sciences, California Institute of Technology, Pasadena, California 91109.

Received 5 March 1969. mological problem. The composition, origin, and subsequent modification of these visible manifestations of differentiation are not independent of the composition and temperature of the primordial preplanetary material. The evolutionary sequence-nebulae to protoplanet to planet to planetary differentiation and outgassing, which the earth has undergone-leads to the origin of life somewhere along the line, presumably during the last stage, which is still in progress. It is not necessarily true that all the planets in the solar system have reached the last stage or that they are all at a similar stage in their evolution. It is not even necessarily true that a planet must eventually reach the point where it extensively differentiates and outgasses. Just as geology, as we know it on earth, has been controlled by events at depth and in the past, so the possibility of life evolving on a planet depends, presumably, on the planet having reached a critical stage in its evolution. Although the chemical and environmental prerequisites for the origin of life are now thought to be understood, these prerequisites have not been fitted into any scheme of planetary evolution.

\section{Boundary Conditions}

The following data are relevant to the present thermal state of the earth and to its thermal evolution:

(1) the "age" of the earth $\sim 4.5 \times 10^{9}$ years;

(2) the oldest known rock $\sim 3.5 \times 10^{9}$ years;

(3) the oldest known rocks are metamorphosed sediments, implying water at the surface of the earth at this time;

(4) oldest rocks with primary remnant magnetism $\sim 2.7 \times 10^{9}$ years;

(5) heat flow through the surface of the earth $\sim 1.2$ $\mu \mathrm{cal} / \mathrm{cm}^{2} \mathrm{sec}$; 
(6) average radioactivities of crustal and upper mantle rocks;

(7) molten rocks come from crust and upper mantle;

(8) region of mantle from $50 \mathrm{~km}$ to $150 \mathrm{~km}$ partially molten to explain seismic low velocity, high attenuation zone;

(9) remainder of mantle is below the melting point, i.e., completely solid;

(10) outer core is primarily iron and is molten;

(11) inner core is primarily iron and is solid.

In addition, it is worthwhile pointing out that the present surface temperatures of the earth are buffered by the $\mathrm{H}_{2} \mathrm{O}$ system, upper mantle temperatures are buffered by the melting point of wet silicate rocks, and that the present composition of the atmosphere is determined to some extent by the presence of life and atmosphere, hydrosphere, crustal interactions, particularly with regard to $\mathrm{H}_{2} \mathrm{O} \mathrm{CO}$, and $\mathrm{O}_{2}$. It should also be pointed out that $\mathrm{H}_{2} \mathrm{O}$ is trapped in the atmosphere by the socalled cold trap, which suppresses the possibility of photodissociation and subsequent escape of hydrogen. These later points are pertinent when one compares the composition of the atmospheres of the earth, Venus, and Mars. Differences in planetary atmospheres could represent primitive differences in the composition of these planets, differences in the processes and degree of outgassing, or simply be a result of meteorological processes traceable ultimately to differences in distance to the sun, rotation rates, inclination of the rotational axis to the plane of the ecliptic, and the presence or absence of a shielding magnetic field.

Even if the temperature were known as a function of depth throughout the earth, it would be impossible to determine the temperatures as a function of depth throughout geologic time since the heat flow equation can only be integrated forward in time. In particular, the present state of the earth cannot be used to deduce its initial state or temperature. However, theories concerning the early state of the earth must be consistent with the above boundary conditions. Temperatures must be high enough to melt iron in the outer core and to melt partially the silicates in the upper mantle but cold enough in the lower mantle and inner core to be below the melting point of the material in these regions. Since the magnetic field of the earth originates in fluid motions in the conducting core, the core must have formed in the first 1.5 billion years of the earth's life, implying that the melting point of iron is exceeded in this time interval. On the other hand, surface temperatures must be suitable for the existence of liquid water before 3.5 billion years ago. These conditions all argue for relatively hot temperatures early in the history of the earth and a relatively rapid cooling off. Since radioactive heating and heat transfer by conduction are slow processes, one concludes that initial heat, i.e., heating due to gravitational accretion, and heat transfer by convection are likely to be important. Unfortunately, most discussions of the thermal history of the earth consider only radioactive heating and conduction limited cooling.

\section{Geophysical Evidence}

The major subdivisions of the earth are the crust, mantle, and core. Rocks as old as 3.5 billion years are known in the continental crust. By contrast the oceanic crust is very young and, in fact, new oceanic crust is being continually created at the oceanic rises by the eruption of basalts. The continents appear to be permanent features even though erosional forces are continually removing material to the continental shelf and ocean basins. To maintain the continental freeboard against the destructive forces requires that new crustal material be added from below or from the sides. Both the oceanic and continental crust are very mobile, and relative motions between the various blocks are now fairly well documented. It is these relative motions, which can be as great as $10 \mathrm{~cm} /$ year or even greater, that are responsible for the major portion of the earth's seismicity. Earthquake belts tend to occur at the contact of the large crustal plates. The rejuvenation of continental crust, the continuous generation of oceanic crust and the extreme mobility of the outer layer of the earth are indications that the earth is far from being a static planet.

The chemical and density stratification of the earth indicates that it is a strongly differentiated body, and there is ample evidence that most of the mass transport involved in the stratification occurred in the liquid phase. The low-melting fraction of the upper mantle is less dense and rises to form crust by igneous and volcanic processes thereby effectively removing heat from the upper mantle. The heat producing radioactive elements are preferentially concentrated in the melt and are therefore also concentrated in the crust and depleted in the mantle. Any volatiles in the mantle are also concentrated in the melt, and this is probably the main mechanism of outgassing. The latent heat of fusion, and the efficient removal of heat and heat sources by the rising magma, serves to buffer the temperature of the upper mantle and keep it near the solidus rather than rising to the point where the upper mantle is entirely molten.

In addition to the volcanic evidence, there is other evidence that the upper mantle is partially molten. From about $50-\mathrm{km}$ to $150-\mathrm{km}$ depth, the velocity of seismic waves is less than in the adjacent levels of the earth. The decrease in velocity is too great to be the effect of a high temperature gradient overwhelming the pressure gradient. One or two percent melt is adequate to explain the low velocities. Seismic waves, especially shear waves, are highly attenuated in this zone, which also suggests partial melting. Inferred temperatures in this region of the mantle are less than the melting point of dry silicate rock, but a small amount of water can lower the melting point by the required amount. Thus, the earth still retains some volatiles in the interior and has not been completely outgassed. The alternative is that crustal rocks containing water or hydrated minerals have been recirculated into the mantle.

There is additional evidence that the temperature gradient in the upper mantle is close to the melting point gradient. The melting temperature of dry silicate 


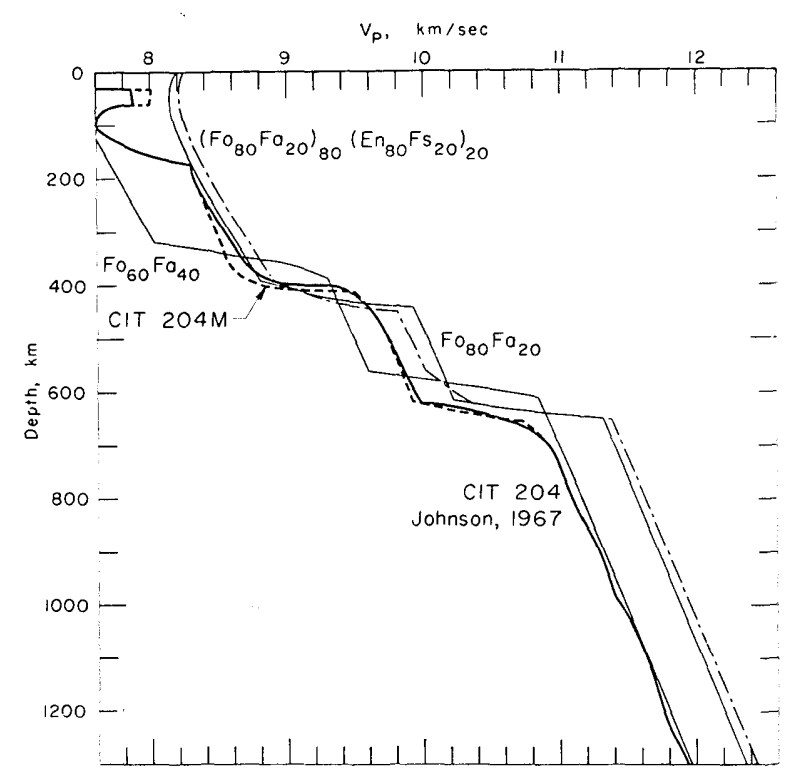

Fig. 1. Compressional velocity $V_{p}$ as a function of depth in the upper mantle of the earth (heavy solid and heavy dashed lines). The light solid lines are two theoretical mantles composed of pure olivine of different compositions. The dash-dot line is for a mantle composed of $80 \%$ olivine $\left(\mathrm{Fo}_{80} \mathrm{Fa}_{20}\right)$ and $20 \%$ pyroxene $\left(\operatorname{En}_{80} \mathrm{~F}_{\mathrm{S}_{20}}\right)$.

rocks and rocks containing small amounts of water is about $10^{\circ} \mathrm{C} / \mathrm{kbar}$. If the amount of melt is small, say $2 \%$ or less, upward transfer of heat by magma motions is restricted and the main heat flow will be by conduction. This gives a heat flow through the upper mantle of about $0.6 \mu \mathrm{cal} / \mathrm{cm}^{2}$ sec. In tectonically inactive regions such as stable shields, heat flow values of about $1 \mu \mathrm{cal} / \mathrm{cm}^{2}$ sec are measured at the surface of which about half can be accounted for by heat generated in the crust by radioactive decay. Thus, a temperature gradient in the upper mantle close to the melting point gradient is consistent with observed heat flow. In volcanic and tectonic regions, heat flow is often greater than $3 \mu \mathrm{cal} / \mathrm{cm}^{2}$ sec and, in these regions, the low velocity or partial melt zone is observed to come nearer to the surface. The consequent upward warping of the isotherms due to upward migration of magma, a convective process, gives larger heat flows in these areas. A partially molten, weak layer in the upper mantle that is effectively decoupled from the adjacent mantle helps to explain the extreme mobility of the outer layers of the earth.

The fact that the outer core is liquid now implies that it settled to the center of the earth in the liquid state, taking its latent heat with it. The temperature gradient in the outer core is presumably adiabatic. The solid inner core can be a result of the melting point gradient being shallower than the melting point gradient or can represent freezing of an initially completely fluid core. In the latter case, the size of the inner core is controlled by the rate at which the latent heat of fusion can be conducted through the core mantle boundary.

The mantle above some $50 \mathrm{~km}$ and below some 150 $\mathrm{km}$ is completely solid. This is known since both shear waves and compressional waves propagate through these regions. The outer core does not transmit shear waves; this is part of the evidence that it is fluid.

Thus, seismology has established that the mantle is solid, the upper mantle is partially molten and presumably very weak, the outer core is molten, and the inner core is solid. These facts have bearing both on tectonic processes, seismicity, the mobility of the outer layers of the earth, and on the evolution of the earth.

In addition to establishing the state of the earth's interior, seismology, combined with ultrasonic and shock wave data, also establishes the composition of the layers of the earth that are inaccessible to more direct sampling. Conventional seismic techniques supply the velocity of propagation of compressional and shear waves. Free oscillation and surface wave studies also provide estimates of density in the various regions of the interior, and these data supplement gravity data, which provide estimates of density in the uppermost mantle. Knowledge of both the density and the elastic properties considerably restricts the range of rock types and compositions that can occur in the upper mantle.

Figure 1 shows the variation of compressional velocity vs depth in the earth as determined by seismological techniques. Note the upper mantle low velocity zone and the two transition regions near $400-\mathrm{km}$ and $600-\mathrm{km}$ depth. As mentioned previously, the low velocity zone is most probably the result of partial melting. Partial melting lowers the density as well as the velocities, making this region of the mantle mechanically unstable. Upward migration of the melt in this region, one possible manifestation of this instability, leads to volcanism or igneous intrusion. This is apparently happening at the midoceanic rises where new crustal material is being formed. Material removed vertically by this mechanism can be replenished laterally by migration of magma in the low velocity layer. If this lateral migration is inhibited, then the crest of the rise will migrate in order to tap an undepleted reservoir. The new oceanic crust, even after it solidifies, is quite mobile, and it migrates away from the rise apparently by sliding on the lubricated surface of the partial melt zone. Small, shallow earthquakes near the crest of the rise accompany the initial breakup.

Down thrusting of the denser outer layers of the earth is the alternate manifestation of instability, and this seems to be occurring at some continental margins, island arcs, and trenches. Most of these features occur around the margin of the Pacific basin and are accompanied by large shallow earthquakes and smaller deep focus earthquakes. Thus, the mass motions due to gravitational differentiation are also responsible for a large fraction of the earth's seismicity and tectonic activity.

The transition regions in Fig. 1 are due to the collapse of normal silicates to high pressure phases. These phase changes are temperature and pressure dependent, and their location in the mantle provides the best method for estimating temperatures at great depth. The dominant mineral of the mantle is olivine $(\mathrm{Mg}$, $\mathrm{Fe})_{2} \mathrm{SiO}_{4}$, a solid solution of forsterite $\left(\mathrm{Mg}_{2} \mathrm{SiO}_{4}\right)$ and 
fayalite $\left(\mathrm{Fe}_{2} \mathrm{SiO}_{4}\right)$. Petrological and seismic evidence suggests that the composition of the olivine in the upper mantle is forsterite, $80-90$ mole $\%$ and fayalite, $20-10$ mole $\%$, or $\mathrm{Fo}_{80} \mathrm{Fa}_{20}$ to $\mathrm{Fo}_{90} \mathrm{Fa}_{10}$. The other main minerals of the mantle are pyroxene [end members enstatite $\left(\mathrm{MgSiO}_{3}\right)$ or En and ferrosilite or $\mathrm{Fs}$ ] and garnet. Figure 1 also shows the variation of $V_{p}$ with depth for two compositions of olivine taking into account temperature, pressure, and composition. Iron rich olivines have a lower $V_{p}$, a higher density, and transform to denser phases at lower pressures. The actual velocities and densities of the various regions of the mantle and the locations of the transition regions provide information about the composition and temperature of these regions.

The $400-\mathrm{km}$ transition region is primarily due to the collapse of the olivine to a spinel structure which is about $10 \%$ denser. The deeper discontinuity is due to the further collapse of the spinel structure to a structure having approximately the density of the close packed oxides $\mathrm{FeO}, \mathrm{MgO}$, and $\mathrm{SiO}_{2}$ (stishovite) which is about $20 \%$ denser than olivine. Similar phase changes occur in pyroxenes and garnets. The dashed curve is a theoretical mantle composed of $80 \%$ olivine and $20 \%$ pyroxene, both containing 80 mole $\%$ of the magnesium rich end member. Note that the iron content of the mantle increases progressively with depth. This is also evidence of differentiation. Either the upper mantle was derived from the lower mantle thereby enriching the lower mantle in the denser elements, or the lower mantle was enriched in iron during the core formation event. In either case the lower mantle, and therefore the earth, is more enriched in iron than had been previously thought.

The triple role of seismology has been alluded to in the previous paragraphs. Namely, it provides data pertinent to the state (molten, solid, partially molten), composition, and tectonics or seismicity of the planet. All of these have bearing on the evolution of the planet. A seismically inactive planet is probably also a relatively undifferentiated planet. We shall discuss later the role that the mass of the planet plays in both the level of differentiation and seismicity. The more massive planets are able to trap more gravitational energy and are more likely to have been extensively differentiated and outgassed.

\section{The Evolution of an Earth-Type Planet}

Prior to the discovery of radioactivity it was generally believed that the earth formed hot, even molten, and has been cooling off ever since. Radioactivity provided a means for a planet to accrete from cold particles and to warm up gradually, and this is the generally accepted view today. This view was strengthened by the observation that present day heat flow from the earth's interior is almost exactly what it would be in the steady state if the earth had the radioactivity of chondritic meteorites. This left very little room for any contribution from initial heat. It has recently been found that meteorite abundances are not representative of mantle rocks and also that many oceanic and con- tinental heat flow values are affected by recent volcanism or igneous activity and cannot be considered steady state values.

The following sequence of events seems to have led up to the present observed situation. The protoplanetary nebulae through magnetohydrodynamic instabilities broke down into regularly spaced preplanetary dust gas accumulations. These concentrations of matter accreted into the planets and satellites. The relative importance of electrostatic, viscous, and gravitational forces were probably different at different stages of the accumulation. The initial sizes of the solid grains is unknown, but gravitational forces would probably not be important until at least one of the bodies in the protoplanetary cloud was roughly moon-sized. The initial temperature of the particles was controlled by solar radiation heating due to collisions and, if the particles were large enough and formed early enough by radioactive decay, particularly that due to short-lived radioactivities. The temperatures were probably of the order of hundreds of Kelvins. Thus the planets can be considered to have accreted from cold particles. Since the atmospheres of the planets are considered, on abundant evidence, to be a result of outgassing of the interior, the primitive particles must have retained large amounts of volatiles and to have been, therefore, relatively cold. The accretional process probably started relatively slowly and accelerated with time as the mass of the solid body increased; gravitational accretion increases as the square of the mass of the accreting body. Accretion will slow down, of course, as the available material in the vicinity of the growing planet is used up. Several lines of geochemical evidence suggested that the accretional process took on the order of $10^{8}$ years, the so-called formation interval. However, if the earth started accreting $4.5 \times 10^{9}$ years ago the earth will stay below the melting point of iron, a prerequisite for core formation, for the first $2 \times 10^{9}$ years. If, in addition, the primitive particles are completely oxidized then surface temperatures must exceed the reduction temperature of iron oxides and iron silicates at the surface of the planet during a sufficient interval of accretion to account for the amount of iron presently in the core. The reduction is presumably due to $\mathrm{H}_{2}, \mathrm{C}$, or $\mathrm{CO}$ present in the primordial cloud. If free iron is present in the accreting particles, as it is today, then it can be incorporated into the growing planet without recourse to this latter mechanism.

If all of the gravitational energy of accretion is retained by the planet then very large initial temperatures will result, as shown in Fig. 2. Even the moon will exceed the melting temperature of silicates during accretion. However, if the planet accretes slowly enough, some of the heating of the surface due to the infall of new material will be compensated for by the radiation of heat to outer space either directly or through radiative and convective processes in the primitive atmosphere. If the planet is growing in radiative equilibrium then the surface temperature is controlled by the equation

$$
[g(r) M(r) / r] \mathrm{d} r / \mathrm{d} t=\epsilon \sigma T^{4},
$$




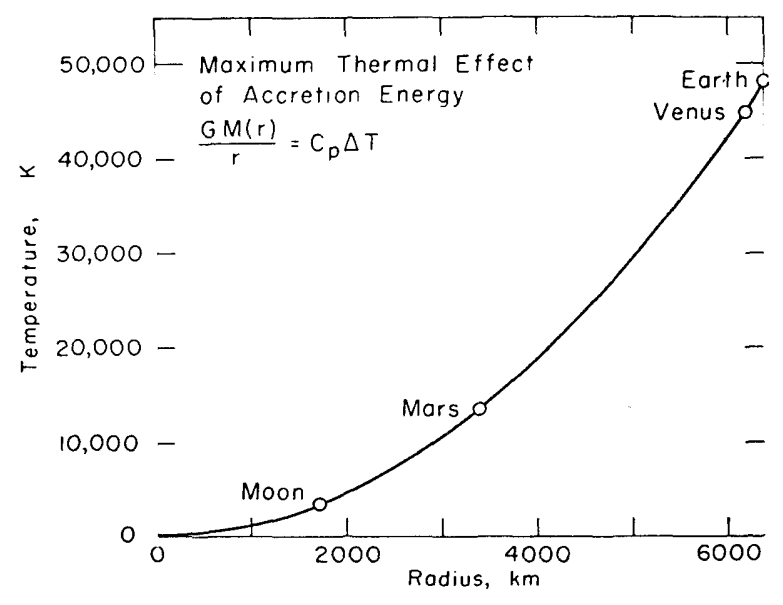

Fig. 2. Maximum temperatures obtained if all of the gravitational energy of accretion is retained by the planet (after Hanks and Anderson, Ref. 2).

where $g$ is the gravitational acceleration, $\mathrm{d} r / \mathrm{d} t$ is the rate of growth, $M$ is the mass below radius $r, \epsilon$ is the opacity of the atmosphere, $\sigma$ is the Stefan-Boltzmann constant, and $T$ is the absolute temperature. For an earth-sized planet accreting uniformly in $10^{8}$ years, the surface temperature during the terminal stages of accretion reaches $250 \mathrm{~K}$. For a more realistic accelerated accretion model, surface temperatures reach $1100 \mathrm{~K}$ for the earth, $110 \mathrm{~K}$ for the moon, and $330 \mathrm{~K}$ for Mars. For these time scales then the terrestrial planets are relatively cold during most of their accretion and the thermal energy required for melting, differentiation, and tectonic processes must be provided by radioactive heating at a later stage of evolution. An additional rise of temperature in the interior is due to adiabatic compression, but this is a relatively small effect.

Once a planet is assembled under the above considerations it will be relatively uniform in composition, both in major elements and in the radioactive heat sources. The temperatures in the deep interior will be less than the temperatures near the surface because of the greater kinetic energy of in-falling particles during the later stages of accretion on a massive planet. Subsequent heating due to radioactive decay must cause substantial amounts of iron to be melted before $2.7 \times 10^{9}$ years ago, and this will initially occur in the outer parts of the planet. Although at zero pressure iron has a slightly higher melting point than silicates, the effect of pressure is to reverse this trend at relatively shallow depths unless the silicates contain substantial amounts of water. In any event the melting of iron will occur first in the upper several hundred kilometers of the earth and the process of core formation may begin. The gravitational energy associated with core formation amounts to about $600 \mathrm{cal} / \mathrm{g}$ or enough to raise the temperature of the earth by an average of $2000^{\circ} \mathrm{C} .1$ This is much greater than the latent heats of fusion of iron and silicates and suggests that the core formation process will be self-accelerating and probably extremely rapid on a geological time scale. This process was discussed in some detail by Birch. ${ }^{1}$ As pointed out by Birch the core formation event was the most profound event in the history of the earth and may, in fact, represent the age of the earth. Because of the large thermal shock of this event it is unlikely that surface rocks could have survived and therefore it probably occurred more than $3.5 \times 10^{9}$ years ago.

However, as mentioned previously, with our present estimates of radioactivity it will take $2 \times 10^{9}$ years for the earth to heat up to the melting point of iron if it accreted in radiative equilibrium in $10^{8}$ years. Either initial temperatures must be higher, implying a more rapid accretion, or radioactivities must be much greater. There is reason to believe that our present estimates of the radioactivity of the earth are already too high. By contracting the time scale of accretion to the order of $10^{5}$ years it is possible to start core formation at an acceptable time after accretion. Geologically this is an extremely short period of time and, since it is an upper bound, the process of core formation could be occurring simultaneously with the accretional process. Figure 3 shows initial temperatures and temperatures at later periods of time for an earth which has accreted in $1.7 \times 10^{5}$ years. Note that when the earth is onethird to one-half of its present size, surface temperatures are large enough to reduce some of the silicates and oxides, thereby producing, for example, free iron. The inner part of the earth or the whole of a much smaller planet will be oxidized, if the original particles are oxidized, and will retain any volatiles present. Outgassing, differentiation, and many chemical reactions will be occurring near the surface during a large part of the accretion of an earth-sized planet.

Various lines of evidence suggest that the accretion was taking place in a dense dust gas cloud and that this cloud and the early volatile products of accretion were blown away or removed by some unspecified process perhaps involving an early, more luminous stage, of the sun. The subsequent outgassing of the deep interior of the planet, the portion that remained relatively cool during planetary assembly, gave rise to the present atmosphere. The planet, of course, must be large enough to maintain this atmosphere against thermal escape.

Thus, the time scale of accretion and the ultimate size of the body are critical in discussions of the differentiation and outgassing of a planet. The first step in differentiation probably involves the melting of iron

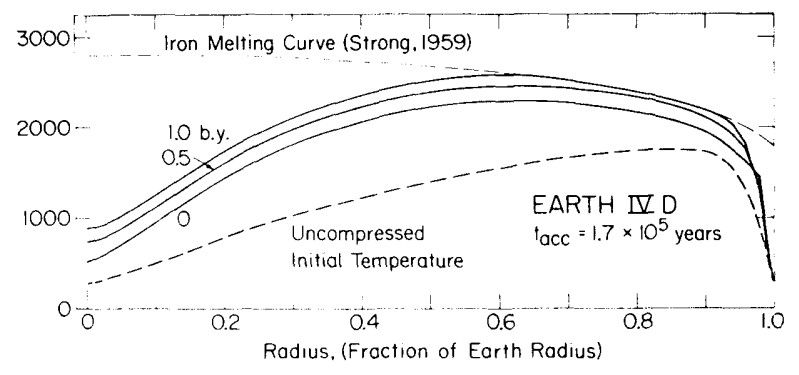

Fig. 3. Incompressed, or surface, temperatures as a function of radius, initial temperatures taking into account accretional energy and adiabatic compression, and temperatures at $0.5 \times 10^{9}$ years and $1.0 \times 10^{9}$ years after accretion (after Hanks and Anderson, Ref. '2) 


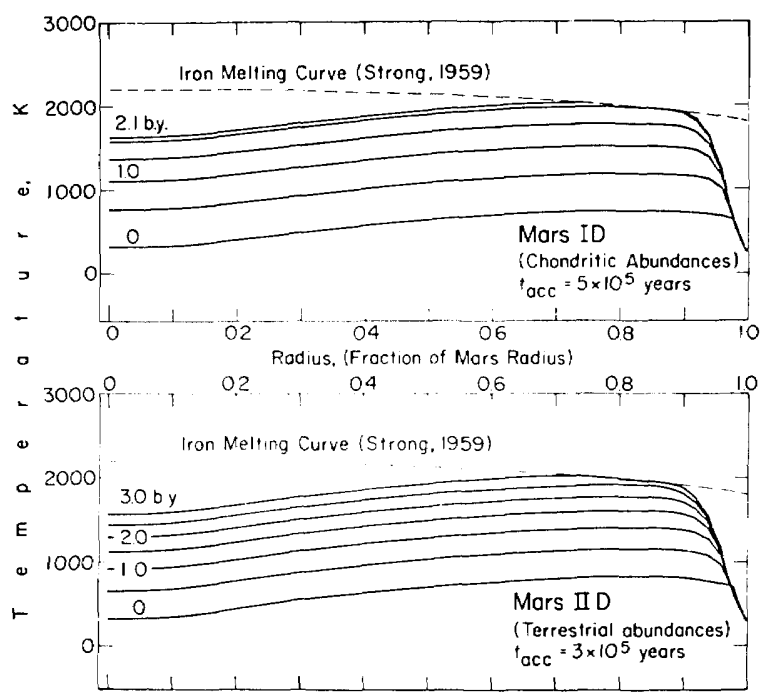

Fig. 4. Initial temperatures and temperatures as a function of time for Mars models having different radioactive abundances (after Hanks and Anderson, Ref. 2).

and the formation of a core. The formation of a large core supplies a large amount of thermal energy and differentiation of the mantle and crust, and outgassing of the atmosphere and hydrosphere were probably triggered by this event. The presence of a core and a crust in a planet is therefore of fundamental geophysical importance and is intimately related to the problem of the development of an atmosphere and a hydrosphere.

\section{Venus, Mars, and the Moon}

The mean density and total mass of Venus suggest that it is very nearly earth-like although it is slightly lighter implying that it has more volatiles or less iron. Since blackbody temperatures in the vicinity of Venus are slightly greater than in the vicinity of the earth the former possibility is less likely although other considerations also apply. The apparent absence of water in the atmosphere of Venus, if due to cosmological rather than meteorological effects, would also be an argument against the increased retention of volatiles in Venus.

The orbital parameters of Venus are enough different from the earth to make one suspect that meteorological phenomena may be responsible for the differences in atmospheric water content. The inclination of the rotation pole of Venus makes seasonal ehanges much less pronounced, i.e., the northern and southern hemispheres will behave symmetrically. The low rotation rate will emphasize differences between the hemispheres facing toward and away from the sun and one can expect large transfer of atmospheric heat and matter between these hemispheres. Atmospheric tides, solar wind intensities, and the absence of a magnetic field on Venus are also pertinent to the evolution of the atmosphere and the escape of atmospheric material. If the $\mathrm{CO}_{2}$ locked up in carbonate rocks were returned to the atmosphere and if the earth were placed in the orbit of Venus with Venus' orbital parameters, would the surface temperature rise by several hundred degrees and would water dissociate and $\mathrm{H}_{2}$ escape from the top of the atmosphere?
This meteorological question should be answered before profound cosmological significance be attached to the observed differences in the atmospheres of these two planets. If the water cannot be trapped in Venus' atmosphere, as it is the earth's atmosphere, then a sink must be found for the resulting oxygen. Such a sink must involve large scale overturning of the surface of Venus or the intrusion of reduced material from depth. The high surface temperatures of Venus may be due to a buffering chemical reaction involving $\mathrm{CO}_{2}, \mathrm{O}_{2}$, and silicate rocks.

Venus is obviously extensively outgassed, but since we do not know its moment of inertia we cannot speculate on the presence of a core. The absence of a magnetic field suggests it either does not have a core or is rotating too slowly to generate and sustain the necessary dynamo action. It is also possible that the magnetic field periodically disappears as it does on the earth and the present situation is only transient. The probability of observing a planet when its field is reversing is, however, very small. Since the similarities between Venus and the earth are more striking than the differences, it is probable the Venus has an earth-like iron rich core but that the slow rotation rate or even perhaps the absence of a sizable satellite or precession of the pole of rotation are responsible for the absence of an internally generated magnetic field.

Mars is much smaller than the earth and on any theory must have been much colder after accretion. The mean uncompressed density of Mars is less than either the earth or Venus suggesting that it retained volatiles, contains less iron, or is in a higher oxidation state. Figure 4 shows one model for the thermal evolution of Mars. The possibility of core formation occurs very late in the history of the planet and the radioactive abundances need be reduced by only $20 \%$ to completely avoid melting of iron. If reduction of iron oxides and silicates is a prerequisite to core formation, i.e., if there is no free iron in the protoplanetary material, then Mars will not satisfy the conditions for core formation even with the increased radioactive abundances.

There are three weak arguments against the presence of a large molten iron core in Mars. The moment of inertia of Mars implies that it is a relatively homogeneous body. Taking into account the increase with depth of density due to compression and solid-solid phase changes there is room for only a very small central core, $300 \mathrm{~km}$ in radius or less. The absence of a magnetic field suggests that Mars has no core or that it is too small to generate a magnetic field. Again, however, the role of a large satellite or of precession are largely unknown. The absence of a shielding magnetic field incidentally has bearing on the origin and evolution of life. The thin atmosphere also suggests that outgassing and, therefore, differentiation has not reached a very advanced stage. The problem of atmospheric escape from a small planet, however, is also pertinent to this observation.

The 12-km relief of Mars, recently discovered by radar measurements, could be used as an argument that Mars has a relatively thick crust and has therefore been 
differentiated. This is basically an argument based on analogy with the earth since large scale relief on the earth is a result of variations in crustal thickness due to collisions of oceanic and continental blocks and to lateral irregularities in the concentration of material differentiated from the mantle. The alternate view is that the surface relief on Mars is due to original inhomogeneities and these are maintained by stresses at depth. If the upper hundreds of kilometers of Mars are much colder than the corresponding region of the earth, these stresses could be maintained for long periods of time. Because of the smaller mass of Mars larger topographic variations can be maintained with a given strength or a given viscosity than on the earth. Only seismic data and measurements of the surface composition would distinguish between these two possibilities. Precise orbital data would also be helpful in determining the density contrast, if any, and the means of support of thes: topographic features.

The moon is a much less massive body than the others discussed and will be intrinsically colder in its early history unless it formed near the earth and was somehow able to share the earth's accretional energy. However, the average radioactivity of the moon is probably greater than the other planets discussed since it is deficient in iron. Both on the earth and in meteorites iron is largely free of the radioactive elements, and the presence of iron in a planet will tend to dilute the radioactivity. The average density of the moon is very close to the average density of the earth's mantle and, for lack of a better criteria, can be assumed to have the same concentration of radioactivities. Alternatively, it can be taken to have the radioactivity abundances of chondrites. In either case the moon will exceed the melting point of silicates in less than $4.5 \times 10^{9}$ years and will produce basalt magmas, if the composition of the moon is similar to the composition of the earth's mantle. The moon, therefore, is also a differentiated body although in a different sense than Venus or the earth, since no iron and, therefore, no core is involved in the differentiation.

This research was supported by NASA Research Grant NGL-05-002-069.

This paper represents Contribution 1620 of the Division of Geological Sciences of the California Institute of Technology. The author wishes to acknowledge the collaboration of $\mathrm{T}$. Hanks in portions of this research.

\section{References}

1. F. Birch, Geol. Soc. Am. Bull. 76, 133 (1965).

2. T. Hanks and D. L. Anderson, Phys. Earth Planet. Interiors 2,19 (1969).

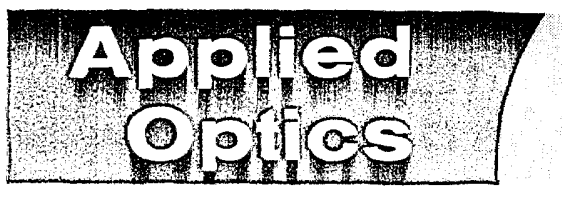

\section{Of Optics and Opticisis}

This column is compiled partly from information sent in by APPLIED OPTICS Reporters in various centers of optics across the world, but the Editorial Consultant welcomes news from any source. It should be addressed to P. R. WAKELING, WINC, 1619 Nineteenth Street N. W., Washington, D.C. 20009

This month we are reprinting in this column, by permission of the Editor of Nature, an editorial that appeared in the April 5 issue of that publication. The editorial discusses a report of a panel, chaired by S. Passman, on which the Editor of Applied Optics served and complements two editorials in this journal.

\section{The Shadow Literature}

Each branch of intellectual life has a body of literature more or less to itself, and none is more correctly organized than the scientific literature. So much is almost self-evident. By common consent, more or less the same rules apply to the 30,000 journals which are now thought to be published in the sciences. Over the years, techniques for simplifying the retrieval of articles in the journals have been worked out. Most journals operate some kind of review system, so that the manuscripts submitted for publication are not automatically turned into type. Publication in almost any journal implies some degree of authenticity for a piece of scientific research. For that reason, and also because of the way in which publications may frequently help in strengthening a person's claim on money or even promotion, it is not surprising that the integrity of the scientific literature is jealously watched over by most working scientists. This is why it is inevitable that a good many thorny issues should have been raised by the study of some of the peripheral parts of the literature, and in particular the technical reports produced by public and industrial laboratories, which has been carried out by the Com- mittee on Scientific and Technical Information (COSATI) in the United States. In the circumstances, it is also understandable that the report on the project, now published by the Federal Council for Science and Technology in Washington, should be a firm call for compromise.

COSATI is chiefly concerned with an attempt to decide what policies on the production of technical reports should be pursued by the agencies which ultimately pay for their production, usually governments. Should the authors of reports be choked off, and persuaded to publish in the conventional scientific journals? Or should governments go the other way, recognize the convenience and often the cheapness of technical reports, and encourage their technical people to produce technical reports and nothing else? The committee's recommendation is neither one extreme nor the other, but an intermediate course which is barely distinguishable from the present state of affairs. This is why the most interesting parts of the report are to be found in the argument by which the committee arrives at its conclusions.

There is no doubt of the speed with which the technical literature has grown. The committee estimates that several hundreds of thousands of new technical reports are being circulated each year in the United States. The Federal Clearing House for Scientific and Technical Information formally announces something like 30,000 new reports a year, and this does not count the very large numbers of technical documents which never see the light of day for reasons of secrecy, military or commercial. The literature of the technical reports is also exceedingly diverse. 